\title{
Deformable printed circuit boards that enable metamorphic electronics
}

\author{
Shantonu Biswas, Andreas Schöberl, Mahsa Mozafari, Jörg Pezoldt, Thomas Stauden and Heiko O Jacobs \\ A method to produce single-layer deformable and stretchable printed circuit boards is reported and applied to enable the \\ realization of metamorphic electronic products that can take on new three-dimensional (3D) shapes. The models contain arrays \\ with packaged surface mount devices and bare dies that integrate light-emitting diodes (LEDs) and transistors within a rubber \\ matrix. The test structures morph from planar to spherical to cone-like topologies. In one approach, the thickness of the \\ stretchable printed circuit board is locally adjusted to control the morphological changes. In addition, a three-dimensionally- \\ shaped chaperon is introduced to enable more abrupt topological changes. A comparative study of various designs of the \\ metallization layer and stress-relieving reinforcing elements identified a highly stretchable and deformable design (up to $315 \%$ or \\ six thousand $150 \%$ stretch and release cycles), which shields the interface between the hard and non-stretchable components \\ from high levels of stress.
}

NPG Asia Materials (2016) 8, e336; doi:10.1038/am.2016.186; published online 9 December 2016

\section{INTRODUCTION}

The field of stretchable and deformable electronics has seen a rapid increase in research activities in recent years. These materials enable new types of applications, such as smart clothing, ${ }^{1,2}$ conformable photovoltaics, ${ }^{3-5}$ optoelectronics, ${ }^{6-11}$ digital cameras, ${ }^{12,13}$ artificial electronic skins, ${ }^{14-16}$ stretchable batteries, ${ }^{17-20}$ robotics, ${ }^{21}$ and mechanically soft and conformable health-monitoring devices, ${ }^{22-26}$ to give a few recent examples.

A pioneering contribution to this field came with the introduction of stretchable conductors by Lacour and Wagner in 2002,27,28 who reported a gold-coated polydimethylsiloxane surface with wrinkles that could be stretched. Subsequently, Gray et al. ${ }^{29}$ reported stretchable meander- or horseshoe-shaped conductors, which are extensively used today. John Rogers and co-workers applied the two basic concepts of Wagner (wrinkled metallic structures) ${ }^{27}$ and Chen (meander-shaped metallic structures $)^{29}$ to thin single-crystal semiconducting films to produce transferable and stretchable semiconductor devices. This research has led to several interesting publications. ${ }^{3-13,19,21-26}$

From a technical point of view, a classification into two fabrication strategies can be made. The first strategy uses a rubber support toward the beginning of the processing sequence. Direct-write or transfer techniques are subsequently used to deposit the required functional materials. Most of the reported models used this method. ${ }^{3-11}$ This approach, however, is challenging in cases when the realization of high-performance devices, and complex circuits or systems is required. Necessary registration and high-temperature processing steps are typically not compatible with the rubber-like support.

The second strategy delays the use of the rubber support for as long as possible. Far fewer reports exploit this strategy. ${ }^{30,31}$ However, there are several advantages of this approach, for example, well-established processing methods can be used. In principle, the entire range of established materials, devices, components, interconnecting strategies, high-temperature processing techniques, precision alignment steps and registration processes can then be used.

This publication reports a method to produce single-layer stretchable printed circuit boards for metamorphic electronics. Unlike most ${ }^{3-11,32,33}$ reports, our approach delays the use of the stretchable rubber support until the end of the processing sequence. Specifically, the entire circuit containing interconnects, unpackaged chips or chipscale packaged surface mount devices are fabricated on a hard carrier. This approach facilitates high-temperature processing, automated mounting and precision alignment. Moreover, our approach enables 'on-hard carrier' functionality tests, which are critical for determining the correct functionality and for comparing the performance metrics after the circuit is released. The application of the rubber support and the release and stretchability tests are conducted last.

The method is used to prepare several test structures. A comparative study of various designs of the metallization layer and stress-relieving reinforcing elements is presented. The reinforcing elements provide critical stress relief in the contact pad region that connects the devices where defects occurred. In combination with computational analysis of the stress profiles, we identified a highly stretchable design to shield the hard and non-stretchable objects from high levels of stress. The newly presented meander design is optimized to distribute and lower the peak stress value and torque in the locations where premature failure commonly occurred. This approach delays the point of failure to extreme levels of elongation, which exceed $315 \%$ or after approximately six thousand $150 \%$ stretch and release cycles. 

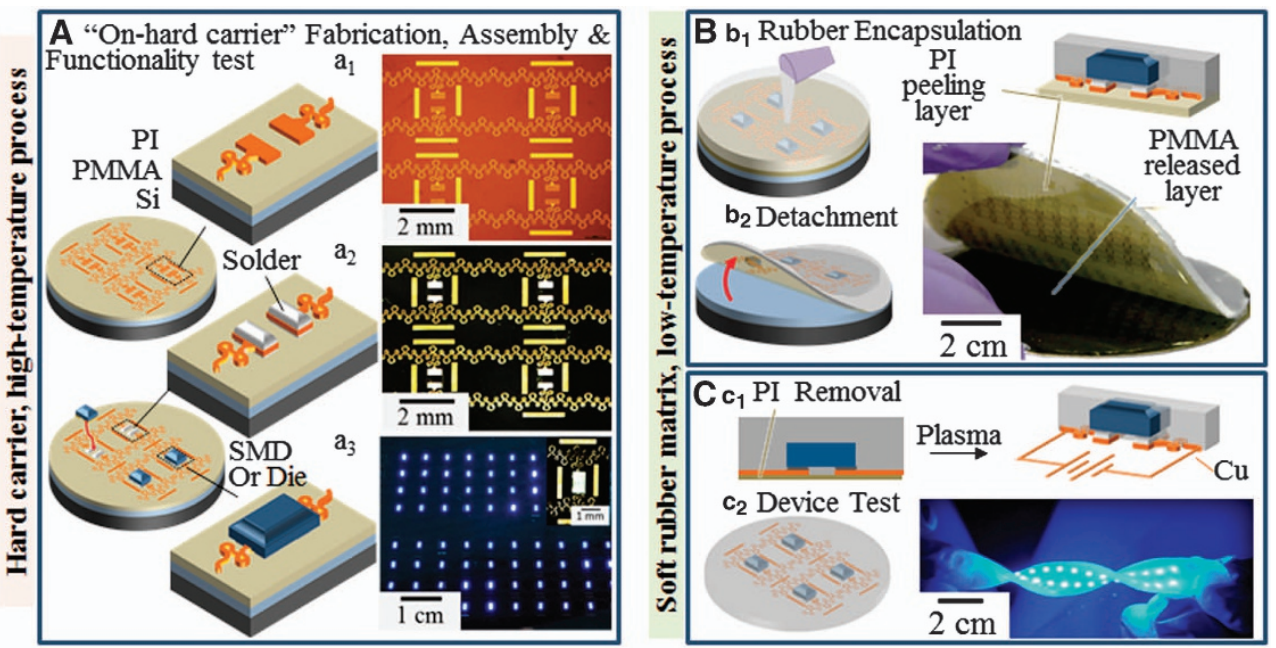

Figure 1 Schematic of the stretchable printed circuit boards process whereby the introduction of the rubber substrate is delayed toward the end of the processing sequence and an increased level of fixation of the parts and metallization layer compared with reversed 'on-rubber' mounted structures. (A) 'Onhard carrier' fabrication, assembly and functionality test. $\left(a_{1}\right)$ PI peeling (greenish gray) layer, metallization (orange), $\left(a_{2}\right)$ solder bumps, $\left(a_{3}\right)$ die attachment and on-hard carrier functionality tests follow the standard PCB (printed circuit board) fabrication, alignment, mounting and device-testing procedures. (B) Rubber matrix application $\left(b_{1}\right)$ and detachment $\left(b_{2}\right)$; the PI peeling layer (green) is used as a common non-stretchable film-like support during the peeling process. The PMMA (poly(methyl-methacrylate)) remains on the hard carrier. (C) Removal of the non-stretchable PI peeling layer ( $\mathrm{c}_{1}$ ), which leaves all faces surrounded by EcoFlex with the exception of the metallization layer, which can be contacted from the bottom $\left(\mathrm{c}_{2}\right)$.

As an application, the concept of metamorphic electronics is demonstrated. Our models are inflatable electronic structures that contain arrays with packaged surface mount devices, bare dies of lightemitting diodes (LEDs) and transistors within a rubber matrix. The test structures morph from planar to spherical to cone-like topologies.

\section{MATERIALS AND METHODS}

Figure 1 provides an overview of the main process steps to realize stretchable printed circuit boards (stretchable PCBs) next to photographs of the corresponding test structures (a more detailed description and flow charts of the process are provided in the supplemental section Supplementary Figure S1). The depicted approach is different from other methods; ${ }^{3-11}$ other methods apply the metallization to the rubber support and mount the components on top, and they suffer from a lower level of alignment and fixation. Instead, a hard carrier is used (A), and mounted components and metal tracks are fixed and surrounded with EcoFlex (B), and subsequently peeled off to complete the device (C). The hard carrier (A) is important because it enables the following: (i) alignment and registration, (ii) high-temperature processing, (iii) conventional robotic chip placement or (iv) advanced chip placement of microscopic dies using fluidic self-assembly, ${ }^{30,34}$ and (v) 'on-hard carrier' device tests.

Figure 1A describes the first part of the processing sequence. The process starts with the application of a polyimide (greenish gray; PI) peeling layer $\left(a_{1}\right)$, which supports the buildup of the circuit $\left(a_{2}, a_{3}\right)$. The peeling layer acts as a mechanical support during the detachment process (B) and is only removed after the fabrication of the stretchable circuit is completed (C). A spin-coated 8 - $\mu \mathrm{m}$-thick layer of polyimide (PI 2611, HD Microsystem, Neu-Isenburg, Germany) on top of a poly(methyl-methacrylate) (PMMA)-coated (AR-P 6510, Allresist, Strausberg, Germany) Si wafer is used. The function of the peeling layer is to provide a mechanically flexible but not stretchable peeling foil, which has a low adhesion to the PMMA-coated (blue) carrier (gray) and a strong and adjustable adhesion to the metal, circuit elements and rubber matrix on top. The required adhesion to the rubber matrix and metallization layer is achieved by plasma activation of the polyimide surface (30 SCCM (standard cubic centimeters per minute) $\mathrm{Ar}, 100 \mathrm{~W}$ for $2 \mathrm{~min}$ ).

$\left(\mathrm{a}_{1}\right)$ As a metallization layer, we used a 20-nm/200-nm-thick sputter-coated layer of $\mathrm{Ti} / \mathrm{Cu}$. This seed layer was patterned by photolithography, and $10 \mu \mathrm{m}$ of electroplated $\mathrm{Cu}$ was added to increase the mechanical robustness of the metal tracks. Thick metal tracks ( $>5 \mu \mathrm{m}$ ) were found to be more robust than the previously used thin $(<1 \mu \mathrm{m})$ metallization layers. ${ }^{30}$ Our attempts to produce a model using thin metallization layers failed. The measured electrical resistance of the 68-mm-long electroplated $\mathrm{Cu}$ tracks was in the range of 2.4-2.8 $\Omega$; this value gives an average resistivity of $1.91 \mu \Omega \mathrm{cm}$ (assuming an average cross-section of $500 \mu \mathrm{m}^{2}$ ), which is very close to the commonly reported bulk value of $1.68 \mu \Omega \mathrm{cm}$. An additional annealing step for $3 \mathrm{~h}$ at $350^{\circ} \mathrm{C}$ under nitrogen had no effect on the already small resistance, and no influence on the subsequent processes was noted. As a result, pristine and otherwise untreated electroplated copper was used in the experiments.

The process is compatible with various types of chip attachment and assembly methods, including solder bump-based interconnects, flip-chip die attachment, robotic pick-and-place or engineered self-assembly using molten solder. In the demonstrated case, a semi-automated pick-and-place machine is used to assemble the components under a microscope in combination with solder bumps to achieve alignment.

A set of lithographic steps involving registration is required to define the locations of the solder bump-based interconnects $\left(a_{2}\right)$; we used a patterned $10-\mu \mathrm{m}$-thick photoresist layer (AZ $15 \mathrm{NXT}$, blue layer) with openings to the contact pads to apply the depicted solder bumps using a dip-coating process in a liquid solder bath. ${ }^{35}$ In the depicted model, two terminal chip-scale ( $L=1 \mathrm{~mm}, W=0.6 \mathrm{~mm}, H=0.2 \mathrm{~mm}$ ) surface-mount LEDs ( $459 \mathrm{~nm}$, Creative LED GMBH, Schaan, Fürstentum Liechtenstein) were used. Specifically, the LEDs are placed on the solder bumps, which are located on the receiving substrate. The reflow of the solder causes the chips to attach and self-align as a result of the reduction of the surface free energy of the solder.

The approach enables 'on-hard carrier' functionality tests $\left(a_{3}\right)$. The illustrated result shows the testing of an array containing 80 LEDs. The 'on-hard carrier' functionality test can be compared with the function after the release and during the operation of the stretchable circuit, which is important to establish and overcome failure modes.

Figure 1B describes the rubber encapsulation and detachment process. We used a castable 3-mm-thick and thermo-curable (room temperature, $15 \mathrm{~h}$ ) layer of EcoFlex (Smooth-On, EcoFlex 00-30) as a stretchable encapsulation layer $\left(\mathrm{b}_{1}\right)$. A 5-min-long $\mathrm{O}_{2}$ plasma activation step for the assembled circuit elements was used to increase the bond strength to the EcoFlex. The plasma activation step reduced the onset of cavitation and delamination during stretching.

A solvent-free detachment process $\left(b_{2}\right)$ is used to detach the components and stretchable circuit from the hard carrier and to increase the range of devices and components that can be used. For example, the plastic package of the LED 


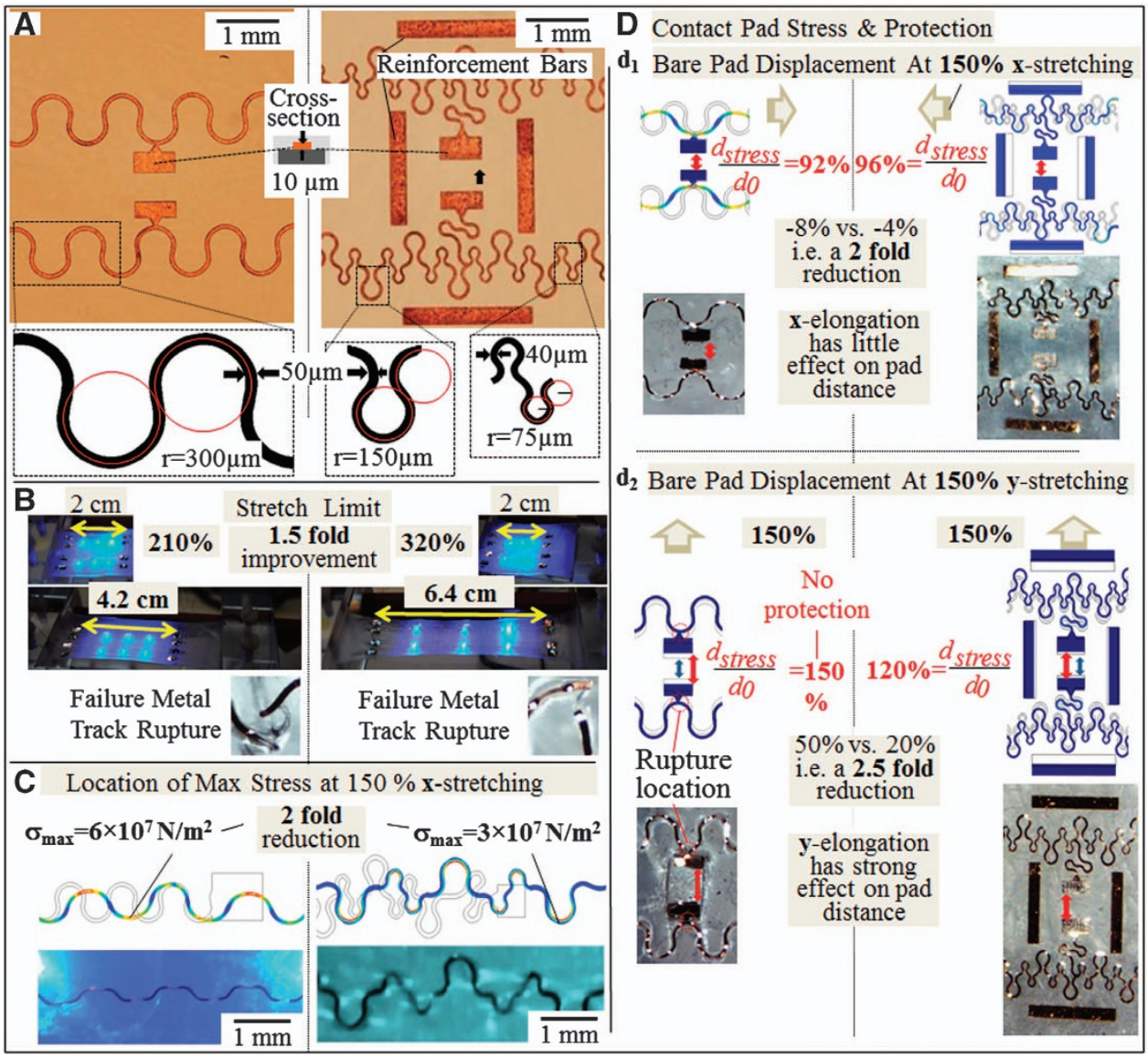

Figure 2 Comparison of two metallization layer designs, stretch limit, location of maximum stress and level of strain relief in the contact pad region: existing (left) and new design (right). (A) Photo of a reference design without strain relief (left) and an improved design that involved altering the width and adding reinforcement bars in critical locations (right). (B) Last video frame of functioning light-emitting diode (LED) array stretched to $210 \%$ (left) and $320 \%$ (right) before failure occurred; the metal tracks rupture beyond this limit (inset). (C) Computed stress profile at a $150 \% x$ axis stretch; the peak stress value is reduced by a factor of 2 in the improved design; the computation and photographs depict the onset of out-of-plane flexing and torsion. (D) Computed and measured contact pad displacement with and without reinforcement bars at a $150 \%$ stretch along the $x\left(d_{1}\right)$ and $y$ axes $\left(d_{2}\right)$. The $y$ axis elongation $\left(d_{2}\right)$ is far more critical; here, the pad distance and strain follow the external strain, and no strain protection is recorded in the reference design (left). By comparison, the reinforced structure (right) leads to a 2.5 -fold strain reduction.

gets damaged in acetone, which is commonly used to remove PMMA. Instead of using a solvent, the detachment process uses the differential interfacial adhesion of the stacked layers to define an interface that can be cleaved. Specifically, the PI (greenish gray) layer has a low adhesion to the PMMAcoated (blue) carrier. Moreover, the rubber matrix is strongly bonded to the PI (greenish gray) layer using the previously mentioned plasma activation step, which leads to the formation of covalent bonds at this interface. As mentioned, the detachment process worked particularly well with the introduced PI (greenish gray) film, which forms a uniform non-stretchable and supporting peeling layer beneath the circuit. The non-stretchable nature and low level of adhesion to the PMMA-coated carrier was critically important to eliminate the introduction of defects during the detachment process. In other words, the sandwich structure is reinforced by the PI film; it is not stretched during the detachment process and no defects are introduced during this step. To illustrate this result, we recorded a video $\left(\mathrm{V}_{1}\right.$, supplemental section) of the detachment process. The video shows the detachment of an LED array while it is being operated. The LEDs continue to light up during the detachment process.

After detachment, the $\mathrm{Cu}$ metal tracks are still covered with the PI peeling foil, which needs to be removed to complete the structure (Figure $1 \mathrm{c}_{1}$ ). We used electron cyclotron resonance plasma etching (40 SCCM O 2,10 SCCM $\mathrm{CF}_{4}, 100 \mathrm{WRF}, 30 \mathrm{~min}$ at $0.0025 \mathrm{mbar}$, SQ160 Roth and Rau AG) to accomplish this step. The final stretchable device has a surface mount-like geometry, which means one of the faces of the copper tracks is accessible for electrical contacts, leaving the other three faces protected with EcoFlex. This arrangement is different from others produced in earlier reports. ${ }^{6-11,24-26}$ In our case, the metal tracks and components are not 'floating' on top of the rubber support. Instead, at least three faces are surrounded by EcoFlex to increase the level of fixation. Moreover, the active devices remain completely embedded in EcoFlex (surrounded and under-filled), which provides protection during plasma etching, processing and the final operation. For example, we characterized the resistance of $68-\mathrm{mm}$-long $\mathrm{Cu}$ metal tracks before and after detachment and plasma etching; the detected increase in the resistance was $9 \%$, and it was predominantly caused by an increase in the contact resistance of the probe needle on the soft substrate (Supplementary Figure S2). This structure was used in the stretch tests discussed below. The increased level of fixation and underfill with EcoFlex in the device region improves the maximum level of elongation before the metal tracks detach from the rubber support, which will be discussed below. It is also possible to fully encapsulate the circuit using a second EcoFlex layer.

\section{RESULTS AND DISCUSSION}

Figure 2 details the design aspects we studied to provide stress relief in critical locations. The first part will focus on the measures taken to provide stress relief in the metal track region (left side, Figure 2) with associated metrics in Table 1A. The second part will focus on the measures taken to provide stress relief in the contact pad region (right side, Figure 2D), with associated metrics in Table 1B. 
Table 1A Study of metal track region

\begin{tabular}{|c|c|c|c|}
\hline & Ref. design & Imp. design & Factor \\
\hline \multicolumn{4}{|l|}{ Single-stretch experiments } \\
\hline \multicolumn{4}{|c|}{ Single-stretch limit to cause a metal track rupture } \\
\hline Elongation limit & $215 \%$ & $320 \%$ & $1.5 \times$ \\
\hline \multicolumn{4}{|c|}{ Single-stretch limit to observe onset of metal track detachment } \\
\hline Elongation limit & $160-180 \%$ & $205-245 \%$ & $1.3 \times$ \\
\hline \multicolumn{4}{|c|}{ Multicycle experiments } \\
\hline \multicolumn{4}{|c|}{ Cycle count at $200 \%$ elong. to cause full metal track detach } \\
\hline Counts & 30 & $>500$ & $>16 \times$ \\
\hline \multicolumn{4}{|c|}{ Cycle count at $150 \%$ elong. to cause metal track rupture } \\
\hline Counts of first conductor to fail & 252 & 3411 & $13 x$ \\
\hline Last conductor to fail & 1312 & 6280 & $5 \times$ \\
\hline Average conductor to fail & 1120 & 5840 & $5 \times$ \\
\hline
\end{tabular}

Abbreviations: imp., improve; Ref., reference.

The presented approach (Figure 2A) uses a variation of the well-established 'horseshoe-shaped conductors', which were originally reported by Gray et al. ${ }^{29}$ Unlike the original design (A, left), our meander-shaped metal ribbons had varied widths and radii of curvature (A, right). Moreover, reinforcement bars were introduced to provide stress relief in the contact pad region that connects the devices where defects occurred. The various iterations and optimization steps, which led to the improved design, are presented in the supplemental section (Supplementary Figure S3). The two depicted designs used the same wire length $(68 \mathrm{~mm}$ to span an unstretched distance of $20 \mathrm{~mm}$ ).

As mentioned, unlike the original design (left), our meander-shaped metal ribbons had varied widths and radii of curvature (right). The rational for this design change is as follows: the weakest points in the reference structure are the upper and lower turning points of the meander, which are parallel to the direction of elongation. These locations are exposed to high levels of torque and stress. The torque propagates to these points because of the buildup of higher and lower levels of stress at the outer and inner edges of the relatively wide and uniform meander, and it eventually leads to premature rupture of the metal track in this region. A combination of two measures was used to improve this section (right). Specifically, we reduced the width of the meander and added a small connecting loop in the section that leads to this turning point to minimize the twist.

Table $1 \mathrm{~A}$ and $\mathrm{B}$ provides a summary of the various metrics, which compares the reference with the new design. A computer-controlled stretching platform was used (Supplementary Figure S5) in the longterm cycling test. The metrics are based on the following experiments:

(i) Single-stretch limit to cause a metal track rupture is an experiment depicted in Figure 2B whereby the structure is stretched until the metal track ruptures. The new design sustained an elongation of $315 \%( \pm 12 \%$; elongated by a factor of 3.15$)$ before permanent electrical failure occurred ( $\mathrm{B}$, right). This value is close to the theoretical limit of $340 \%$, considering the ratio between the $68-\mathrm{mm}$ long meander-shaped metal track that connects the 20-mm-spaced (unstretched) contact pads. The established reference structure with a uniform width $(50 \mu \mathrm{m})$ sustained an elongation of $215 \%( \pm 16 \%$, from eight measurements) during the same test (B, left). In both cases, rupture occurred in the upper and lower turning points of the meander. These points are parallel to the stretch direction. A finite element computation (COMSOL Multiphysics) of the stress profile
Table 1B Study of contact pad region

\begin{tabular}{lccc}
\hline Single-stretch experiments & Ref. design & Imp. design & Factor \\
\hline Bare contact pad displacement & without and with reinforce & \\
$x$-Direction & $-8 \%$ & $-4 \%$ & $2 \times$ \\
$y$-Direction & $+50 \%$ & $+20 \%$ & $2.5 \times$ \\
\hline
\end{tabular}

next to a photograph of the test structures stretched to $150 \%$ confirms that the upper and lower turnings are the locations of maximum stress. A reduction of the peak stress value by a factor of 2 is calculated for the improved design ( $C$, right), which explains why it can be stretched to a higher level.

(ii) Single-stretch limit to observe the onset of metal track detachment represents an experiment to study the onset of metal track detachment. A careful observation of both test structures revealed that the metal tracks began to detach from the EcoFlex matrix when the structures are stretched to high levels. We characterized a total of 16 samples (eight each for the reference and new design) with microscopy to record the onset of detachment. The onset occurred between $160-180 \%$ and $205-245 \%$ in the reference structure (left) and improved design (right), respectively. In both structures, detachment started at the locations where the twist in the metal structure is largest. The locations are also the upper and lower turning points of the meander, which are parallel to the direction of elongation. The computation (Figure 2C) provides insight into the reason for this result; again, the detachment and delamination are a result of the buildup of higher and lower levels of stress at the outer and inner edges of the meander, which leads to a flexing and torsion and an eventual detachment of the ribbon-like metal tracks. The detachment of the metal track can thus be understood as a mechanism to provide stress relief; the structure is no longer coupled to the rubber substrate, and a distribution of stress is possible, which reduces the hot spots. However, for real applications, detached metal tracks need to be prevented for several reasons. First, partially detached metal tracks are insufficiently stable for the stretchable PCBs to be touched by hand. Second, the detached region typically grows larger as the number of stretch and release cycles increase. The metal tracks begin to flex and bend into unpredictable directions. Such adverse conditions lead to random arrangements and electrical shorts between the tracks (Supplementary Figure S4). Considering the long-term use, the level of deformation should thus be limited to values where no detachment occurs.

(iii) Cycle count at $200 \%$ to cause complete metal track detachment represents an experiment whereby we determined the number of stretch and release cycles at $200 \%$ elongation until the meander is completely detached from the substrate. It took 30 stretch and release cycles to cause a complete detachment from the substrate in the reference design. The improved design continued to function for 500 cycles $(\sim 1 \mathrm{~Hz})$, after which we aborted the test to study the level of detachment. No detachment from the surface was observed.

(iv) Cycle count at $150 \%$ to cause complete metal track rupture is based on an experiment whereby a larger data set was acquired to determine the average and range between the first and last failure. A single frame and the magnified section of a larger data set are shown in Figure 2C; the full data set and overview picture (Supplementary Figure S6) containing 16 meanders can be found in the supplemental section; each meander spanned an unstretched distance of $20 \mathrm{~mm}$, and one half used the reference structure, whereas the other half used the improved design. A modest $150 \%$ stretch and release cycle was applied in this test. The 'first conductor to fail' failed after 252 and 3411 cycles 


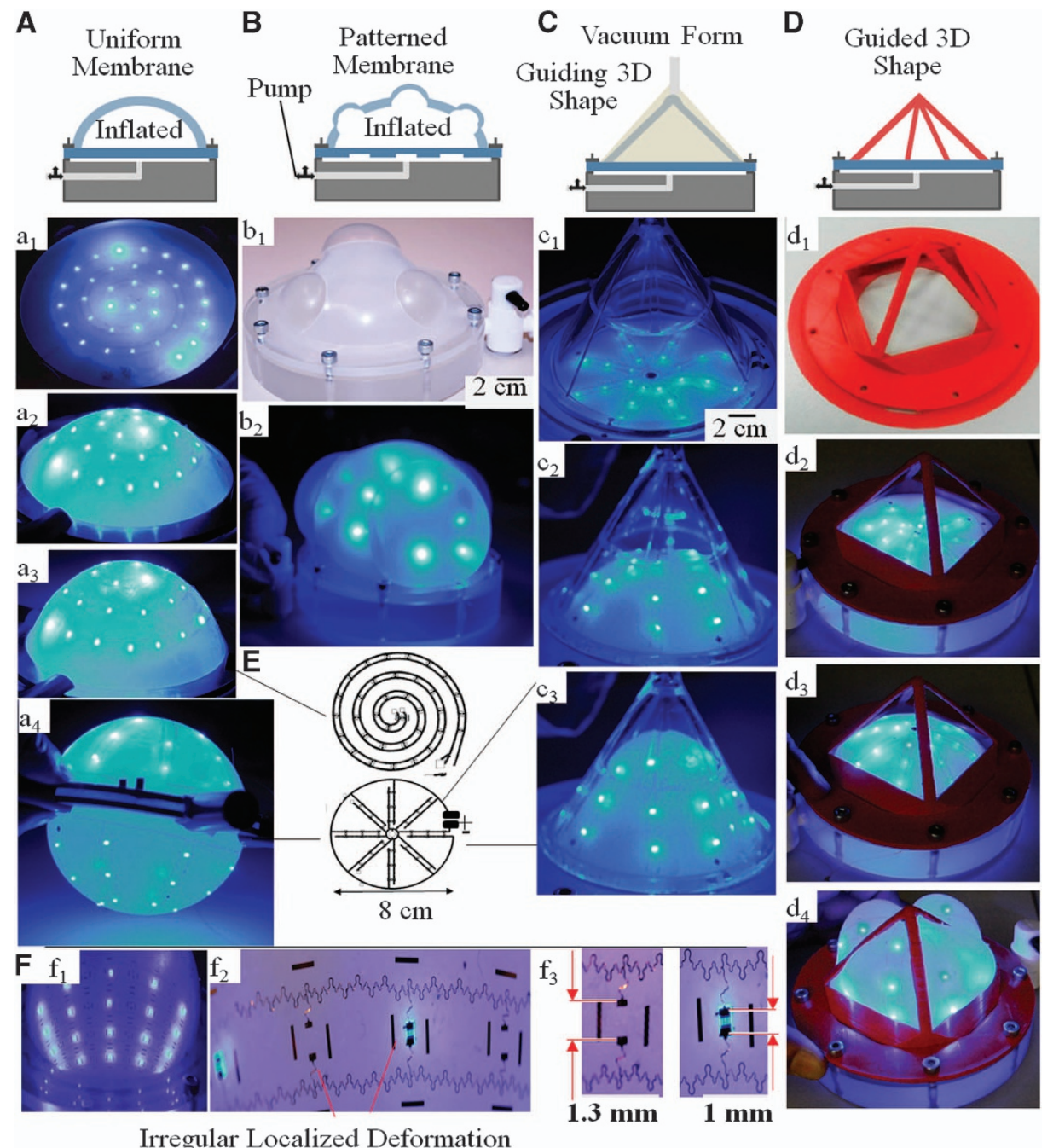

Figure 3 Schematic, experimental results and corresponding CAD designs of inflatable metamorphic lighting structures, which employ a membrane with uniform thickness (A) that morph from planar to hemisphere $\left(a_{1}-a_{3}\right)$, and a sphere $\left(a_{4}\right)$. (B) a membrane with a locally adjusted thickness: without electronics $\left(b_{1}\right)$ and with electronics in operation $\left(b_{2}\right)$. Guiding 3D (three-dimensional) shapes $(\mathbf{C}, \mathbf{D})$ to form a cone $\left(c_{1}-c_{3}\right)$ and a cage $\left(d_{1}\right)$ during inflation $\left(d_{2}-d_{4}\right)$ to control the morphological changes. (E) CAD designs are used for the experiments, and close-ups (F) are used to study the level of strain relief due to the reinforcing structure. This test compares regions with and without assembled LEDs (light-emitting diode) at a $225 \%$ areal stretch.

in the reference and improved design, respectively. In relative terms, the first failure is delayed by a factor of 13.5. The "last conductor to fail' failed after 1312 and 6280 cycles in the reference and improved design, respectively. On average, the occurrence of rupture for the 20-mm-long tracks was delayed from 1120 to 5840 cycles using the improved design. This result represents a fivefold improvement.

Figure 2D presents the reinforcement bars (left) used to protect the contact pad and intermediate surrounding from high levels of stress (right); the corresponding metrics are summarized in Table 1B. The reinforcement bars are used to locally change the material properties of the stretchable PCB by creating reinforced islands where the chips are assembled. Ideally, as the PCB is stretched globally, the islands are not stretched. Consequently, the (i) shear force acting on the assembled components, (ii) lateral pull on the contact pads and (iii) force acting on the metal track connection can be reduced. Among the three, the force acting on the metal track connection is most critical as the metal track connection to the pad fails first in our experiments. The ideal design (different from what is shown) would be a sufficiently thick non-stretchable frame, such as a frame created using a second patterned metallization layer underneath the assembled chips. However, even with a single metallization layer, the reinforcement bars can be defined and located in the vicinity of the components to offer some protection. For example, experimentally, we find that the reference design (left) without reinforcement bars is sensitive to fail if it is elongated in the $y$ direction. Specifically, the intermediate metal track connection to the pad (red circle) fails because of rupture at elongations that exceed $200 \%$, and this value is improved to $325 \%$ using the reinforcement bars; the supplemental section (Supplementary Figure S7) provides an arrangement of images, which support this observation.

(v) Bare contact pad displacement without and with reinforcement bars represents an experiment to study the level of strain reduction in the pad region and intermediate surrounding as a result of the reinforcement bars. The results are shown in Figure 3D, which presents computation data (COMSOL Multiphysics) next to photographs of the stretched structures. The data compare the displacement of the contact pads with and without reinforcement bars as the structure is stretched to $150 \%$ of the original length along the 


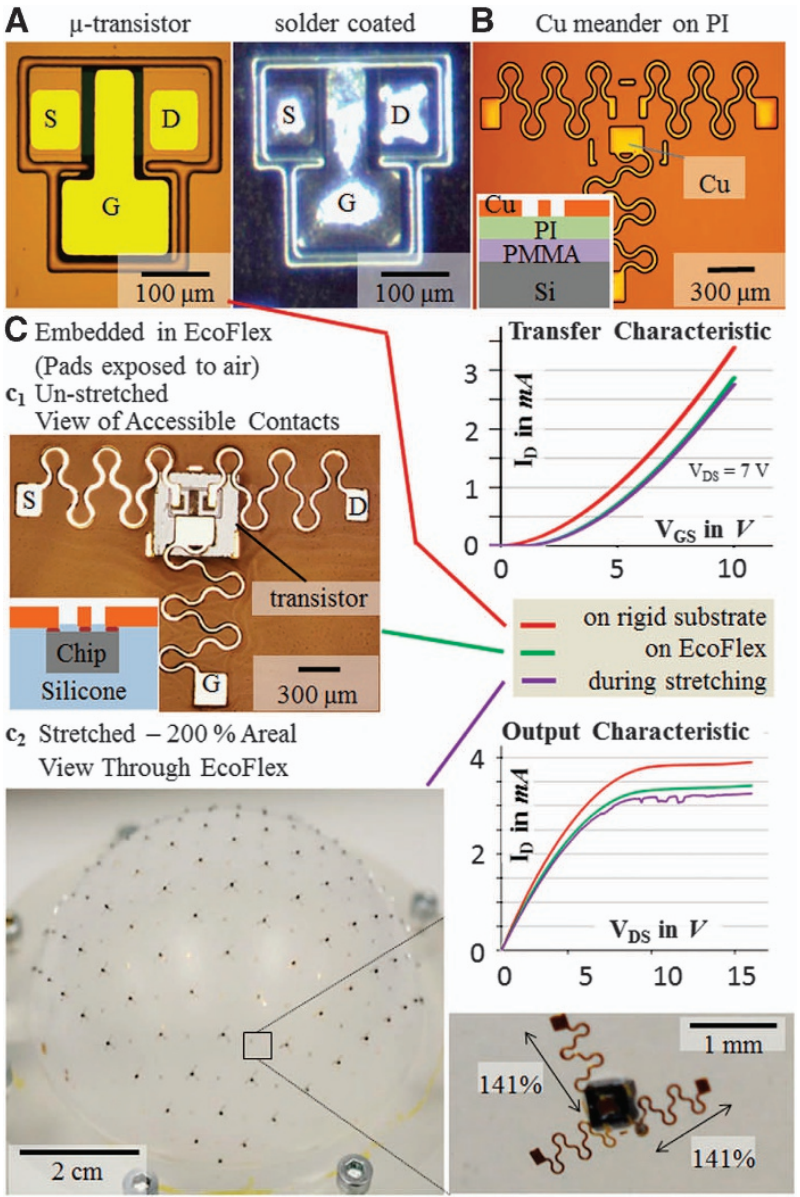

Figure 4 Hemispherical transistor array with 500- $\mu \mathrm{m}$-sized chips. (A) Fabricated transistor before (left) and after (right) solder coating, before dicing the wafer. (B) Photo of the stretchable connections on top of the PI peeling foil before flip chip mounting of the transistors. (C) Photographs of the chips and interconnects embedded in EcoFlex in the unstretched condition $\left(c_{1}\right)$ and during inflation to a $200 \%$ areal stretch $\left(c_{2}\right)$; the chips are immersed in EcoFlex (insert, $c_{1}$ ); the Ti-coated (mirror looking, $c_{1}$ ) bottom surface of the $\mathrm{Cu}$ pads is facing the observer and it is accessible. The insets depict the corresponding $I-V$ curves of the transistors.

$x$ axis $\left(\mathrm{d}_{1}\right)$ and $y$ axis $\left(\mathrm{d}_{2}\right)$. Note: the structures were studied without assembled chips to isolate the effect of the reinforcement bars. As the rubber matrix is stretched, the distance between the pads changes; the smaller the change is, the better the level of protection; the distance is proportional to the force that acts on the metal track connection and joints once the chips are assembled. The level of protection due to the reinforcing bars can be observed by comparing the left with the right side.

First, the elongation along the $x$ axis $\left(\mathrm{d}_{1}\right)$ when compared with the $y$ axis $\left(\mathrm{d}_{2}\right)$ is far less critical for the depicted pad layout. Only a small reduction $(-8 \%$, left) in the pad distance, which is barely visible $(-4 \%$, right) in the reinforced design, can be detected as the structure is elongated along the $x$ axis $\left(\mathrm{d}_{1}\right)$. This result indicates that, considering the strain on the pads and connecting wire, both designs are fairly robust when the structures are stretched in the $x$ direction. However, this effect is no longer the case for the $y$ direction $\left(\mathrm{d}_{2}\right)$. For example, in the reference structure (left), the relative change in the pad distance is equal to the relative external elongation, and no protection of the pads and connecting wire can be observed. This effect leads to premature interconnect failure in the pad region once the assembly of a chip results in a fixed pad distance because of high levels of stress. As a result, reinforcing bars are required to maintain a sufficient level of robustness to allow stretching of the structures in the y-direction. For example, in the improved design (right) a 2.5 -fold reduction of the relative external elongation is achieved in the pad region, which enabled the realization of the inflatable metamorphic electronic models discussed below where a biaxial stress resiliency is required.

Figure 3 depicts the schematics and experimental results of inflatable metamorphic lighting structures. The supplemental section provides additional images, Supplementary Figure S8 and a Supplementary Video V2, of the inflation process while the devices are operated. We used a uniform 3-mm-thick layer of EcoFlex in (Figure 3A), a non-uniform 3- and 1.5-mm-thick EcoFlex layer in (Figure $3 \mathrm{~B}$ ), and secondary guiding topologies (a cone and cage) in Figure $3 \mathrm{C}$ and $\mathrm{D}$ to prepare several different topologies. All test structures used a circular polymer ring with a diameter of $4 \mathrm{~cm}$ to form a pressure seal and electrical connections with an opposing surface. The first structure (Figure 3A) contains 35 LEDs and morphs from a planar $\left(\mathrm{a}_{1}\right)$ to a spherical $\left(\mathrm{a}_{2}-\mathrm{a}_{4}\right)$ structure. The second (Figure $3 \mathrm{~B}$ ) contains 24 LEDs and morphs from a planar $\left(\mathrm{b}_{1}\right)$ to a more complex topology with localized hemispherical extrusions $\left(b_{2}\right)$; the third (Figure 3C) contains 24 LEDs and morphs from a planar $\left(c_{1}\right)$ to a hemispherical $\left(c_{2}\right)$ to a cone-shaped $\left(c_{3}\right)$ geometry; the last structure (Figure 3D) uses a cage to define the edges at desired locations. The cone (C) and cage (D) act as a chaperon to control the deformation. Figure $3 \mathrm{E}$ presents the computer-aided design (CAD) layouts of the array design that were used for the models.

The depicted methodology is different from conventional balloon designs where the resting shape resembles the target structure, which is a concept that has been used in the field of stretchable electronics. ${ }^{12}$ Our resting shapes show little similarities with the final structure. For example, the concept depicted in (Figure 3B) uses a rubber membrane where the thickness is adjusted locally to produce a desired three-dimensional (3D) topology. Localized extrusions occur where the membrane thickness is reduced. From a theoretical design point of view, the Laplace pressure equation

$$
P=2 \gamma / R,
$$

can be used to predict the local radius of curvature $R$. The equation provides a relationship between the pressure $P$, the rubber tension $\gamma$ and the localized radius of curvature $R$. Without applied pressure, the membrane stays flat, which represents an infinitely large radius of curvature. As the pressure increases, the membrane bulges, which reduces the radius of curvature $R$. Simultaneously, the tension begins to increase following $\gamma=E \times \Delta l / l_{\mathrm{o}} \times h$ (2), with Young's Modulus $E$, strain $\Delta l l_{\mathrm{o}}$ and membrane thickness $h$. The illustrated structures agree well with the anticipated spherical shapes and radii of curvature over the range of pressure (15-40 mbar above atmospheric pressure, Supplementary Figure S9) we tested, which suggests that the layer of EcoFlex dominates the elastic properties on a macroscopic level.

However, there are local effects due to the reinforcing bars. The images in (Figure 3F) provide an analysis of the local level of elongation using an inflated test structure. The test structure contains locations without (dark) and with (illuminated) assembled LEDs to study the change in the distance between the pads as the structure is stretched. The depicted and global areal increase is $225 \%\left(\mathrm{f}_{1}\right)$, which represents a factor of 1.5 in terms of the biaxial elongation. This global elongation factor can be compared with the local elongation in the contact pad region by comparing locations with and without the assembled chips $\left(\mathrm{f}_{2}\right)$. For example, the distance between the contact 
pads to accommodate the LEDs is $1 \mathrm{~mm}$; this distance is fixed once a chip is assembled even as the surrounding structure is stretched (illuminated regions); as a result, the force will act and be concentrated on the connecting wires and contact pads. Without the assembled LEDs (dark regions), this distance is not fixed but is influenced by the reinforcement bars $\left(f_{3}\right)$. Considering the depicted $1.5 \times$ biaxial elongation, an increase to $1.5 \mathrm{~mm}(+50 \%$, the full amount of the external strain) could be anticipated. However, the localized reinforcing bars reduce the distance between the contact pads to $1.3 \mathrm{~mm}$ ( $+30 \%$, a reduced amount of strain reaches the pad). In other words, the mechanical stress acting on the $1 \mathrm{~mm}$ separated contact pads is relieved as a result of the reinforcing bars. The relative level of relief is roughly a factor of $1.66(0.5 \mathrm{~mm}$ per $0.3 \mathrm{~mm})$. These bars were critical to produce the illustrated defect-free models. Without bars, the connecting wire to the LED pad failed at an earlier time.

The approach is not limited to the assembly of LEDs. Figure 4 shows three terminal field effect transistors, which we fabricated using a conventional CMOS process (the details are provided in the supplement section). Here, the contact pads on the bare transistors were solder-coated (Figure 4A) and mounted on the same Cu-coated PI peeling layer (Figure 4B). The same EcoFlex encapsulation and release process (previously discussed in Figure 1) is used to produce the depicted stretchable assemblies (Figure 4C). The test structure here contains an array with 100 transistors. Similar to the case of the LEDs, the electrical function of the transistors (inset) is not affected, as the matrix is stretched. The insets depict the corresponding transfer and output characteristics. We measured the characteristics under three different conditions: (i) the pristine transistors on the original wafer (red curve) and the transistors embedded in EcoFlex in the (ii) unstretched (green) and (iii) stretched condition (purple). The pristine transistors (A) show a slightly higher current and a smaller threshold voltage than those in the final state $(C)$. The threshold voltage $\left(V_{\mathrm{t}}\right)$ increases from 1.29 to $1.41 \mathrm{~V}$. The threshold voltage was determined by fitting the recorded drain current $I_{\mathrm{D}}$ to the well-established equation $I_{\mathrm{D}}=k / 2 \times\left(V_{\mathrm{GS}}-V_{\mathrm{t}}\right)^{2}$, with $k$ as a constant. ${ }^{36}$ This small increase is a result of the plasma etching step used to remove the PI peeling layer. However, the transistor characteristic is stable once the device is encapsulated in EcoFlex (C), and no changes can be observed, as the matrix is stretched $\left(c_{2}\right)$ to $200 \%$. At present, each transistor is located in an electrically isolated location, and we are unable to test the function of all the transistors within the array. The required routing of 300 connections is presently not possible. A multiplexed circuit and triple-layer routing would be required to access the 100 elements and 300 contact pads, which is a goal for future studies.

\section{CONCLUSION}

In summary, we reported a method to produce single-layer stretchable integrated circuit boards using a process that is fully compatible with conventional printed circuit board technology, commercially available surface mount devices and robotic mounting methods. The comparative study of various designs of the metallization layer and stress relief mechanisms using reinforcement bars helped identify highly stretchable designs, which shield the hard and non-stretchable objects from high levels of stress. The reinforcement bars shift the point of failure away from the solder bump-based interconnects to the stretchable metal track region. This approach delays the point of failure to extreme levels of elongation that exceed $315 \%$ or after six thousand $150 \%$ stretch and release cycles. However, the reinforcing structures come at a price because they increase the area and footprint surrounding the chips, which could present a disadvantage.
A potential solution would be a multilayer approach. In the future, additional advances should be possible using designs that further improve the distribution of the stress in the metal track region. For example, concepts of interfacial slip similar to veins in the human body, which distribute the strain uniformly, could be included.

Various inflatable metamorphic electronic test structures were fabricated. The presented approach to control the morphological changes is different from conventional balloon designs and 3D-shaped membranes, for which the resting shape resembles the target structure $^{12}$ and complex mounting and transfer techniques are required. Instead, the method presented uses planar fabrication techniques. The resting shapes are planar rubber membranes with integrated electronics. The rubber thickness is adjusted locally to control the morphological changes. The metamorphic structures presented to demonstrate this concept are relatively simple, and a wider variety of structures can be envisioned in future. However, this method will eventually have limits, particularly in cases when rapid changes in the topology, strong bending angles or edge-like topologies are desired. More demanding topological changes such as these will require additional mechanical guides. For example, a grid of reinforcing structures could be added, which has not yet been tested. Another potential solution is the inflation inside of a 3D-shaped chaperon to guide the morphological changes. In principle, such guides could be produced using $3 \mathrm{D}$ printers. At present, a simple cone and cage were used to illustrate this idea.

From a technology point of view, the presented approach is currently limited to a single metallization layer. This limits the level of complexity from an electrical routing point of view. For example, the transistors can, in principle, be used to produce a stretchable active matrix array. However, such an array requires at least two stretchable metallization layers with vias in between them. Going forward, research will be required to enable the fabrication of truly multilayer stretchable printed circuit boards. In addition, stress relief and mechanical reinforcement within the 3D multilayer topology would become critically important. However, such a technology, if successful, would enable any electronic product known today to take on new 3D shapes and interesting form factors in the future.

\section{CONFLICT OF INTEREST}

The authors declare no conflict of interest.

\section{ACKNOWLEDGEMENTS}

The research received partial financial support through grants from the German Science Foundation (JA 1023/-1) and the Carl-Zeiss Foundation (0563-2.8/399/1).

1 Zeng, W., Shu, L., Li, Q., Chen, S., Wang, F. \& Tao, X. M. Fiber-based wearable electronics: a review of materials, fabrication, devices, and applications. Adv. Mater. 26, 5310-5336 (2014).

2 Vieroth, R., Loxher, T., Seckel, M., Dils, C., Kallmayer, C., Ostmann, A. \& Reichl, H. in International Symposium on Wearable Computers, 33-36 (IEEE, 2009).

3 Rogers, J. A., Lagally, M. G. \& Nuzzo, R. G. Synthesis, assembly and applications of semiconductor nanomembranes. Nature 477, 45-53 (2011).

4 Lee, J., Wu, J. A., Shi, M. X., Yoon, J., Park, S. I., Li, M., Liu, Z. J., Huang, Y. G. \& Rogers, J. A. Stretchable GaAs photovoltaics with designs that enable high areal coverage. Adv. Mater. 23, 986-991 (2011).

5 Ahn, B. Y., Duoss, E. B., Motala, M. J., Guo, X. Y., Park, S. I., Xiong, Y. J., Yoon, J., Nuzzo, R. G., Rogers, J. A. \& Lewis, J. A. Omnidirectional printing of flexible, stretchable, and spanning silver microelectrodes. Science 323, 1590-1593 (2009).

6 Sheng, X., Robert, C., Wang, S. D., Pakeltis, G., Corbett, B. \& Rogers, J. A. Transfer printing of fully formed thin-film microscale GaAs lasers on silicon with a thermally conductive interface material. Laser Photonics Rev. 9, L17-L22 (2015).

7 Sun, Y. G., Kumar, V., Adesida, I. \& Rogers, J. A. Buckled and wavy ribbons of GaAs for high-performance electronics on elastomeric substrates. Adv. Mater. 18, 2857-2862 (2006). 
8 Sun, Y. G., Choi, W. M., Jiang, H. Q., Huang, Y. G. Y. \& Rogers, J. A. Controlled buckling of semiconductor nanoribbons for stretchable electronics. Nat. Nanotechnol. $1,201-207$ (2006)

9 Sun, Y. G., Kim, H. S., Menard, E., Kim, S., Adesida, I. \& Rogers, J. A. Printed arrays of aligned $\mathrm{GaAs}$ wires for flexible transistors, diodes, and circuits on plastic substrates. Small 2, 1330-1334 (2006).

10 Khang, D. Y., Jiang, H. Q., Huang, Y. \& Rogers, J. A. A stretchable form of single-crystal silicon for high-performance electronics on rubber substrates. Science $\mathbf{3 1 1}$, 208-212 (2006)

11 Kim, D. H., Ahn, J. H., Choi, W. M., Kim, H. S., Kim, T. H., Song, J. Z., Huang, Y. G. Y., Liu, Z. J., Lu, C. \& Rogers, J. A. Stretchable and foldable silicon integrated circuits. Science 320, 507-511 (2008).

12 Song, Y. M., Xie, Y. Z., Malyarchuk, V., Xiao, J. L., Jung, I., Choi, K. J., Liu, Z. J. Park, H., Lu, C. F., Kim, R. H., Li, R., Crozier, K. B., Huang, Y. G. \& Rogers, J. A. Digital cameras with designs inspired by the arthropod eye. Nature 497, 95-99 (2013).

13 Rogers, J. A., Someya, T. \& Huang, Y. G. Materials and mechanics for stretchable electronics. Science 327, 1603-1607 (2010).

14 Someya, T., Kato, Y., Sekitani, T., Iba, S., Noguchi, Y., Murase, Y., Kawaguchi, H. \& Sakurai, T. Conformable, flexible, large-area networks of pressure and thermal sensors with organic transistor active matrixes. Proc. Natl Acad. Sci. USA 102, 12321-12325 (2005).

15 Tee, B. C. K., Wang, C., Allen, R. \& Bao, Z. N. An electrically and mechanically self-healing composite with pressure- and flexion-sensitive properties for electronic skin applications. Nat. Nanotechnol. 7, 825-832 (2012).

16 Wagner, S., Lacour, S. P., Jones, J., Hsu, P. H. I., Sturm, J. C., Li, T. \& Suo, Z. G. Electronic skin: architecture and components. Phys. E 25, 326-334 (2004).

17 Gaikwad, A. M., Zamarayeva, A. M., Rousseau, J., Chu, H. W., Derin, I. \& Steingart, D. A. Highly stretchable alkaline batteries based on an embedded conductive fabric. Adv. Mater. 24, 5071-5076 (2012).

18 Kaltenbrunner, M., Kettlgruber, G., Siket, C., Schwodiauer, R. \& Bauer, S. Arrays of ultracompliant electrochemical dry gel cells for stretchable electronics. Adv. Mater. 22 2065-2067 (2010).

19 Xu, S., Zhang, Y. H., Cho, J., Lee, J., Huang, X., Jia, L., Fan, J. A., Su, Y. W., Su, J., Zhang, H. G., Cheng, H. Y., Lu, B. W., Yu, C. J., Chuang, C., Kim, T. I., Song, T., Shigeta, K., Kang, S., Dagdeviren, C., Petrov, I., Braun, P. V., Huang, Y. G., Paik, U. \& Rogers, J. A. Stretchable batteries with self-similar serpentine interconnects and integrated wireless recharging systems. Nat. Commun. 4, 1543 (2013).

20 Gaikwad, A. M., Khau, B. V., Davies, G., Hertzberg, B., Steingart, D. A. \& Arias, A. C. A high areal capacity flexible lithium-ion battery with a strain-compliant design. Adv. Energy Mater. 5, 3 (2015).

21 Kim, R. H., Kim, D. H., Xiao, J. L., Kim, B. H., Park, S. I., Panilaitis, B., Ghaffari, R., Yao, J. M., Li, M., Liu, Z. J., Malyarchuk, V., Kim, D. G., Le, A. P., Nuzzo, R. G., Kaplan, D. L., Omenetto, F. G., Huang, Y. G., Kang, Z. \& Rogers, J. A. Waterproof AllnGaP optoelectronics on stretchable substrates with applications in biomedicine and robotics. Nat. Mater. 9, 929-937 (2010)

22 Kim, D. H., Lu, N. S., Ma, R., Kim, Y. S., Kim, R. H., Wang, S. D., Wu, J., Won, S. M., Tao, H., Islam, A., Yu, K. J., Kim, T. I., Chowdhury, R., Ying, M., Xu, L. Z., Li, M. Chung, H. J., Keum, H., McCormick, M., Liu, P., Zhang, Y. W., Omenetto, F. G., Huang, Y. G., Coleman, T. \& Rogers, J. A. Epidermal electronics. Science 333, 838-843 (2011)

23 Webb, R. C., Bonifas, A. P., Behnaz, A., Zhang, Y. H., Yu, K. J., Cheng, H. Y., Shi, M. X., Bian, Z. G., Liu, Z. J., Kim, Y. S., Yeo, W. H., Park, J. S., Song, J. Z., Li, Y. H., Huang, Y. G., Gorbach, A. M. \& Rogers, J. A. Ultrathin conformal devices for precise and continuous thermal characterization of human skin. Nat. Mater. 12 , 938-944 (2013).
24 Huang, X., Liu, Y. H., Chen, K. L., Shin, W. J., Lu, C. J., Kong, G. W., Patnaik, D., Lee, S. H., Cortes, J. F. \& Rogers, J. A. Stretchable, wireless sensors and functional substrates for epidermal characterization of sweat. Small 10 , 3083-3090 (2014)

25 Kim, J., Banks, A., Cheng, H. Y., Xie, Z. Q., Xu, S., Jang, K. I., Lee, J. W., Liu, Z. J., Gutruf, P., Huang, X., Wei, P. H., Liu, F., Li, K., Dalal, M., Ghaffari, R., Feng, X., Huang, Y. G., Gupta, S., Paik, U. \& Rogers, J. A. Epidermal electronics with advanced capabilities in near-field communication. Small 11, 906-912 (2015).

26 II Park, S., Brenner, D. S., Shin, G., Morgan, C. D., Copits, B. A., Chung, H. U., Pullen, M. Y., Noh, K. N., Davidson, S., Oh, S. J., Yoon, J., Jang, K. I., Samineni, V. K., Norman, M., Grajales-Reyes, J. G., Vogt, S. K., Sundaram, S. S., Wilson, K. M., Ha, J. S., Xu, R. X., Pan, T. S., Kim, T. I., Huang, Y. G., Montana, M. C., Golden, J. P., Bruchas, M. R., Gereau, R. W. \& Rogers, J. A. Soft, stretchable, fully implantable miniaturized optoelectronic systems for wireless optogenetics. Nat. Biotechnol. 33, 1280-1286 (2015)

27 Périchon Lacour, S., Huang, Z., Suo, Z. \& Wagner, S. in MRS Online Proceedings Library Archive, Vol. 736, 183-188 (Cambridge Univ Press, 2003).

28 Lacour, S. P., Wagner, S., Huang, Z. Y. \& Suo, Z. Stretchable gold conductors on elastomeric substrates. Appl. Phys. Lett. 82, 2404-2406 (2003).

29 Gray, D. S., Tien, J. \& Chen, C. S. High-conductivity elastomeric electronics. Adv. Mater. 16, 393-397 (2004).

30 Vanfleteren, J., Bossuyt, F., Löher, T., Hsu, Y. Y., Gonzalez, M. \& Günther, J. in Stretchable Electronics 207-233 (Wiley, 2013).

31 Park, S. C., Biswas, S., Fang, J., Mozafari, M., Stauden, T. \& Jacobs, H. O. Millimeter thin and rubber-like solid-state lighting modules fabricated using roll-to-roll fluidic self-assembly and lamination. Adv. Mater. 27, 3661-3668 (2015).

32 Vanfleteren, J., Gonzalez, M., Bossuyt, F., Hsu, Y.-Y., Vervust, T., De Wolf, I. \& Jablonski, M. Printed circuit board technology inspired stretchable circuits. MRS Bull. 37, 254-260 (2012).

33 Hirsch, A., Michaud, H. O., Gerratt, A. P., De Mulatier, S. \& Lacour, S. P. Intrinsically stretchable biphasic (solid-liquid) thin metal films. Adv. Mater. 28, 4507-4512 (2016).

34 Park, S.-C., Fang, J., Biswas, S., Mozafari, M., Stauden, T. \& Jacobs, H. O. A first implementation of an automated reel-to-reel fluidic self-assembly machine. Adv. Mater. 26, 5942-5949 (2014)

35 Jacobs, H. O., Tao, A. R., Schwartz, A., Gracias, D. H. \& Whitesides, G. M. Fabrication of a cylindrical display by patterned assembly. Science 296, 323-325 (2002).

36 Sze, S. M., Kwok, K. Ng. in Physics of Semiconductor Devices 3rd edn, Ch. 6, 306 (John Wiley \& Sons Inc., Hoboken, NJ, USA, 2007).

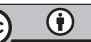

This work is licensed under a Creative Commons Attribution 4.0 International License. The images or other third party material in this article are included in the article's Creative Commons license, unless indicated otherwise in the credit line; if the material is not included under the Creative Commons license, users will need to obtain permission from the license holder to reproduce the material. To view a copy of this license, visit http:// creativecommons.org/licenses/by/4.0/

(C) The Author(s) 2016

Supplementary Information accompanies the paper on the NPG Asia Materials website (http://www.nature.com/am) 